\title{
Comparison of Commercial and Noncommercial Endotracheal Tube-Securing Devices
}

\author{
Daniel F Fisher MSc RRT, Christopher T Chenelle, Andrew D Marchese MSc, \\ Joseph P Kratohvil LPN RRT, and Robert M Kacmarek PhD RRT FAARC
}

\begin{abstract}
BACKGROUND: Tracheal intubation is used to establish a secure airway in patients who require mechanical ventilation. Unexpected extubation can have serious complications, including airway trauma and death. Various methods and devices have been developed to maintain endotracheal tube (ETT) security. Associated complications include pressure ulcers due to decreased tissue perfusion. Device consideration includes ease of use, rapid application, and low exerted pressure around the airway. METHODS: Sixteen ETT holders were evaluated under a series of simulated clinical conditions. ETT security was tested by measuring distance displaced after a tug. Nine of the 16 methods could be evaluated for speed of moving the ETT to the opposite side of the mouth. Sensors located on a mannequin measured applied forces when the head was rotated vertically or horizontally. Data were analyzed using multivariate analysis of variance, with $P<.05$. RESULTS: Median displacement of the ETT by the tug test was $0 \mathrm{~cm}$ (interquartile range of $0.0-0.10 \mathrm{~cm}$, $P<.001)$. The mean time to move the ETT from one side of the mouth to the other ranged from $1.25 \pm 0.2 \mathrm{~s}$ to $34.4 \pm 3.4 \mathrm{~s}(P<.001)$. Forces applied to the face with a vertical head lift ranged from $<0.2$ newtons $(\mathrm{N})$ to a maximum of $3.52 \mathrm{~N}(P<.001)$. Forces applied to the face with a horizontal rotation ranged from $<0.2 \mathrm{~N}$ to $3.52 \mathrm{~N}(P<.001)$. Commercial devices produced greater force than noncommercial devices. CONCLUSIONS: Noncommercial airway holders exert less force on a patient's face than commercial devices. Airway stability is affected by the type of securing method. Many commercial holders allow for rapid but secure movement of the artificial airway from one side of the mouth to the other. Key words: airway holder; endotracheal tube; secure airway; tracheal intubation; mechanical ventilation. [Respir Care 2014;59(9):1315-1323. ( 2014 Daedalus Enterprises]
\end{abstract}

\section{Introduction}

The purpose of an artificial airway is to relieve upper airway obstruction, facilitate suctioning, allow effective ventilation, and prevent aspiration. Unintended removal or dislodgement of the endotracheal tube (ETT) can have

Mr Fisher, Mr Chenelle, Mr Marchese, and Mr Kratohvil are affiliated with Respiratory Care Services, Massachusetts General Hospital, Boston, Massachusetts. Mr Marchese is also affiliated with the Computer Science and Artificial Intelligence Laboratory, Massachusetts Institute of Technology, Cambridge, Massachusetts. Dr Kacmarek is affiliated with the Department of Anesthesiology, Critical Care and Pain Medicine, Massachusetts General Hospital, and Harvard Medical School, Boston, Massachusetts.

Supplementary material related to this paper is available at http://www. rcjournal.com. harmful effects, ranging from localized trauma and aspiration of oral/gastric secretions to death as a result of a compromised airway. ${ }^{1}$ Alternatively, extended pressure

\footnotetext{
This work was funded by a gift from Hollister. Dr Kacmarek received research grants from Covidien, Hamilton Medical, GE Healthcare, Newport, and Dräger and an honorarium for lecturing from Covidien and Maquet and is a consultant for Newport. The other authors have disclosed no conflicts of interest.

Mr Fisher presented a version of this paper at the AARC Congress 2012, held November 10-13, 2012, in New Orleans, Louisiana.

Correspondence: Daniel F Fisher MSc RRT, Massachusetts General Hospital, Blake 652, 55 Fruit Street, Boston, MA 02114. E-mail: dfisher2@partners.org.
}

DOI: $10.4187 /$ respcare.02951 
from securing the ETT on the surrounding tissue can lead to pressure sores and mucosal damage. This is a direct result of the securing device causing pressure points that decrease local tissue perfusion. ${ }^{2}$ In addition, the ETT can be inadvertently advanced to the carina or into a main bronchus.

\section{See the Related Editorial on Page 1456}

The Joint Commission has made pressure ulcer prevention a national patient safety goal (http://www.joint commission.org, Accessed May 2, 2012). Pressure ulcers can range from discomfort to disfiguring sores. Current American Heart Association guidelines suggest that an ETT should be secured with "tape or a commercial device." 3 There are a myriad of devices that are designed to secure an ETT. All of these devices and techniques have similar goals: to keep the artificial airway secure and to keep the patient safe by maintaining an intact airway and minimizing the chance of an unplanned extubation. When considering the type of device to secure the airway, ease of use, efficiency in keeping the airway secure, and ability to reposition the ETT to prevent pressure ulcer formation should be considered. Methods of securing an ETT vary from straps of tape or cotton string to mechanical devices with integrated securing and movement mechanisms. The purpose of this study was to evaluate a wide array of commercially available devices and traditional ETTsecuring techniques under simulated clinical conditions. The conditions tested were designed to evaluate the ability of the holder to keep the ETT in place, the rapidity of relocating the ETT from one side of the mouth to the other, and how much force is transmitted to surrounding areas covered by the device as the head is moved.

\section{Methods}

Sixteen unique ETT-holding devices or ETT-securing methods were subjected to 4 separate tests to evaluate performance in simulated daily activities of the mechanically ventilated, acutely ill patient. The tests performed were: (1) the static tug test, subjecting the ETT secured by one of the holders to a momentary force pulling on the ETT in an attempt to remove the ETT from the airway; (2) ETT movement, determination of the time required to move an ETT from one side of the mouth to the other; (3) vertical movement, determination of the pressure applied by the device to the face and neck during simulated raising and lowering of the head by $30^{\circ}$; and (4) horizontal movement, determination of the pressure applied by the device to the face and neck during simulated turning of the head from side to side in a $70^{\circ}$ arc. All commercial devices were supplied by the manufacturer (Table 1). Some of the

\section{QUICK LOOK}

\section{Current knowledge}

Following placement of an artificial airway, securing the tube to prevent accidental removal or unintended migration is recommended. Unplanned extubation and right main bronchus intubation each have important consequences. Both commercially available and noncommercial devices (eg, adhesive tape) are used.

\section{What this paper contributes to our knowledge}

Commercial airway-securing devices exert greater force on a patient's face than noncommercial devices, possibly increasing the risk of injury. Commercial devices allow more rapid movement of the tube from side to side. Neither type of device is superior in all characteristics and situations.

holders evaluated incorporated a band of material that wrapped around the back of the neck for stability. For those holders, the tension along the band was set to allow 2 fingers to pass snugly between the band and the surface of the mannequin head parallel to the head.

\section{Airway Model}

An anatomically correct adult intubation model (Airway Larry 25000033, Laerdal Medical, Wappingers Falls, New York) was used to simulate the head and upper airway. An 8.0-mm internal diameter ETT (Mallinckrodt Hi-LO, Covidien) was inserted into the trachea and secured by each of the 16 devices/methods evaluated. The cuff was inflated to $25 \mathrm{~cm} \mathrm{H}_{2} \mathrm{O}$, establishing a minimal leak. ${ }^{4}$ Cuff pressure was checked and re-established immediately before each experimental trial.

\section{Static Tug Test}

The intubation mannequin was orally intubated at the beginning of each series of evaluations and secured, in turn, with each device. Before each intubation, the upper airway was lubricated with silicone spray (airway lubricant 252090, Laerdal Medical) to simulate lubrication of the airway with secretions and to allow for greater freedom of movement than was available with dry plastic-onplastic.

The ETTs were secured with each of the 16 devices/ methods. After securing the ETT, the cuff was slowly inflated to a pressure of $25 \mathrm{~cm} \mathrm{H}_{2} \mathrm{O}$ and monitored by a pressure manometer with an incorporated Luer-Lok con- 


\section{COMPARISON OF ETT Holders}

Table 1. Devices/Techniques Used

\begin{tabular}{ll}
\hline \hline & \\
\hline Commercial Device & \\
Ambu ETT holder (with blue strap) 320264040 & Manufacturer \\
Ambu ETT holder (with white strap) 320264041 & Ambu (Glen Burnie, MD) \\
Stabilock ETT holder & Dale Medical Products (Plainville, MA) \\
AnchorFast ETT holder & Hollister (Libertyville, IL) \\
Thomas ETT holder & Laerdal Medical (Wappingers Falls, NY) \\
Marpac 320 ETT holder & Marpac (Albuquerque, NM) \\
Marpac 320 ETT holder with optional head strap & Marpac \\
Portex Quickstrap ETT holder & Smiths Medical (Dublin, OH) \\
ETT holder PM1110 & Precision Medical (Northampton, PA) \\
Cushioned ETT holder 1065 & Teleflex Medical (Research Triangle Park, NC) \\
& \\
Noncommercial Technique & Material Used \\
Clove hitch knot & Cotton twill \\
Cow hitch knot & Cotton twill \\
Rolling (magnus) hitch knot & Cotton twill \\
Hy-Tape & Hy-Tape (one-fourth inch; Hy-Tape International, Patterson, NY) \\
Lillehei method & Cloth tape (Covidien, Mansfield, MA) \\
Modified Lillehei method & Cloth tape (Covidien)
\end{tabular}

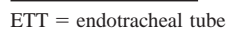

nection (BE 148-7, Instrumentation Industries, Bethel Park, Pennsylvania). Using modified test apparatus as previously described, 5,6 the ETT was connected to a pre-stressed 80pound test nylon line (Mason Tackle, Otisville, Michigan). The opposite end of the line was threaded through a pulley and attached to a 578-g weight. The resulting angle formed between the pulley and the intubation head was $30^{\circ}$, equal to the head-of-bed elevation angle suggested in the ventilator-associated pneumonia bundles (Fig. 1). ${ }^{7}$ A reference mark was made on the side of the ETT denoting tooth position at the beginning of the test.

During a pilot test for this study, the weight was applied without a tug, and tube movement was recorded. No discernable movement occurred. To prevent bias toward adhesives, the face of the mannequin was covered with a new sheet of plastic film (Blenderm, 3M, St Paul, Minnesota) at the start of testing on each new device. This was to prevent any potential interaction between the adhesive and residual cleaner and to provide a better surface for adherence than the outer covering of the intubation head.

The weight was then dropped from a height of $98 \mathrm{~cm}$ and allowed to fall freely before stopping abruptly at $16 \mathrm{~cm}$ above the tabletop, producing a jolt of $\sim 5.7$ newtons $(\mathrm{N})$ on the ETT in the direction away from the mannequin as an attempt to extubate the model. The distance the ETT moved was then recorded. This process was repeated at 5 drops per run and 5 runs per series using 4 devices per evaluation, resulting in a possible 100 drops for each holder evaluated. Following the fifth drop and measurement, the

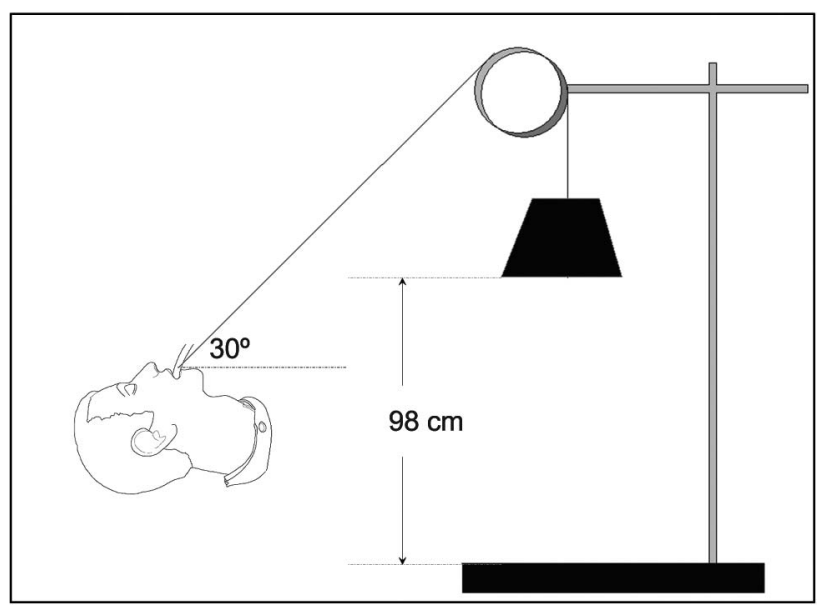

Fig. 1. Static tug setup. The angle between the intubation head and the pulley was $30^{\circ}$, simulating a patient in the Semi-Fowler's position. The weight was dropped and allowed to fall freely until stopping abruptly, causing a jolt.

cuff was deflated, the ETT was repositioned, and the securing method was refastened. At the end of the fifth run in each series, the securing device was removed, the ETT was placed back into the starting position and secured with a new device from the same manufacturer, and the complete series of runs was repeated. A new, previously unused device was used during each series for each securing technique.

If the ETT became dislodged or moved $>5 \mathrm{~cm}$ during testing, it was considered an extubation, and testing was 


\section{COMPARISON OF ETT HoldERS}

stopped for that cycle. (This distance is considerably greater than the $20 \mathrm{~mm}$ suggested by others. ${ }^{6}$ ) The ETT was then repositioned and refastened with a new device/method, and a complete series of drops was repeated with a new device/method. If the ETT was dislodged completely again, the series with the greatest number of measurements was used for analysis. When the distance moved was $<5 \mathrm{~cm}$, this value was recorded, the initial tube position was reestablished, and testing was continued.

\section{Side-to-Side ETT Movement}

For this portion of the evaluation, 6 of these devices were excluded due to a common design feature that prevented lateral tube movement: an integrated bite block. Another device was excluded because it was an optional head strap that would have no impact on lateral tube position. The specific devices excluded from this evaluation were: Ambu holder with Velcro, Ambu holder with a silicone strap, Laerdal Medical Thomas tube holder, Precision Medical ETT holder PM1110, Portex Quickstrap, Teleflex cushioned ETT holder 1065, and Marpac 320 with head strap. The 9 remaining devices/methods were evaluated for speed in moving the ETT from one corner of the mouth to the other. For the commercial devices, the manufacturers' instructions were followed. With the noncommercial methods, the knots evaluated were tied as described by a web-based knot reference (http://www. animatedknots.com, Accessed May 2, 2012). Before testing, the investigator practiced each knot until it could be rapidly tied without assistance.

At the start of the testing, the ETT was secured adjacent to one corner of the mannequin's mouth. The investigator moving the airway signaled to the timing investigator when to start the stopwatch. When the airway was repositioned, the repositioning investigator signaled when to cease timing. This action was repeated 10 times for each method. To limit variation in technique, the same person (DFF) performed all repositioning, and the same person (JPK) kept time with all devices and techniques.

If there was a problem during the movement, if either investigator felt that conditions were not optimal, or if the results were considered to be outside of $1 \mathrm{SD}$ compared with the other trials of the same device/method, the trial was discarded and repeated using the same device/ method.

\section{Head Rotation Study}

The intubation head was removed from the stock torso and placed on a computer-controlled platform with independent motion on 2 axes (Fig. 2). Digital encoders were attached to each drive motor to provide spatial reference for the axis of rotation. Control of each motor was gov-

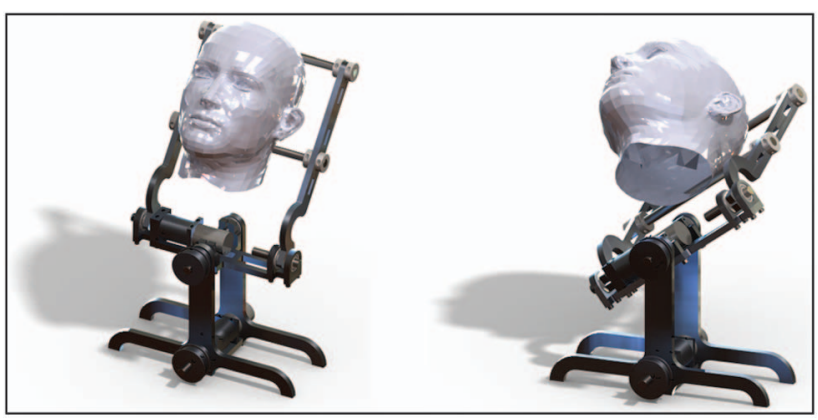

Fig. 2. Illustration of the mannequin setup for the vertical and horizontal movement evaluations. The platform uses gear motors and encoders to pivot the mannequin head in 2 dimensions. Control for the motors is via a Labview interface.

erned via a graphical interface (Labview, National Instruments, Austin, Texas). Five force-sensing resistors (Interlink Electronics, Camarillo, California) were placed around the mouth and nose (Fig. 3). An additional sensor was placed on the back of the neck. Sensor data from the force-sensing resistors were recorded as a voltage every $100 \mathrm{~ms}$ using an analog-to-digital converter and output as a text file. During analysis, the voltage was converted to newtons of force based upon the separate calibration curves for each sensor that were developed before any measurements.

The upper airway was lubricated with silicone spray, and the head was orally intubated in the same fashion as described previously. The ETT was secured using a new device/method for each trial. The secured ETT was then connected to a ventilator circuit (Hudson RCI model 780-32, Teleflex Medical) supported by a crossarm.

The vertical elevation test was carried out by lifting the head from $3^{\circ}$ to $30^{\circ}$, simulating the head being raised from a flat to a semirecumbent position at a rate of 12 rotations/min. For the horizontal rotation testing, the head was moved in a $70^{\circ}$ arc at a rate of 5 rotations $/ \mathrm{min}$. The run time for each axis was 10 min to allow for stabilization of the system. Due to the design features of each ETTsecuring device/method, the force-sensing resistors were moved under potential high-pressure points as determined by the investigators during pilot studies. Specific sensor locations for each device are provided (see the supplementary materials at http://www.rcjournal.com).

The system was started with the head in the neutral position, $0^{\circ}$ elevation and $0^{\circ}$ rotation from midline. During this startup, the system would recalibrate position. Testing occurred only with one axis movement at any time. Data were recorded at a rate of $100 \mathrm{~ms}$, which included all sensor voltages, angular positions, and times. This recording was saved in a text file for later analysis. 


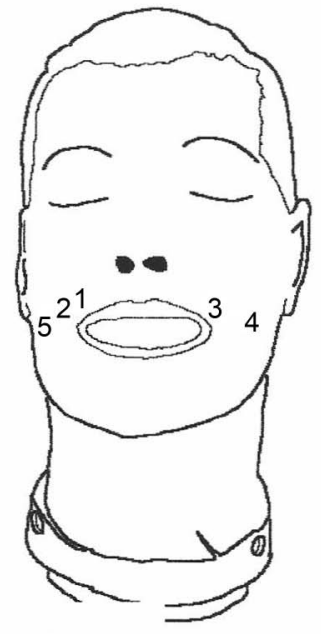

6 Back of neck

All knots

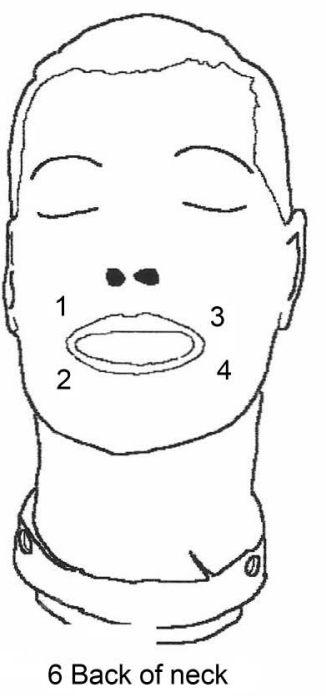

Lillihei

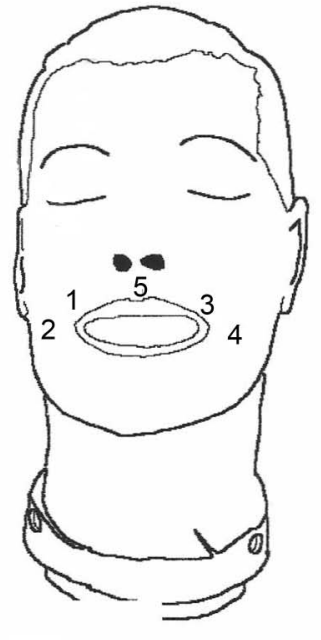

6 Back of neck

Dale/Marpac

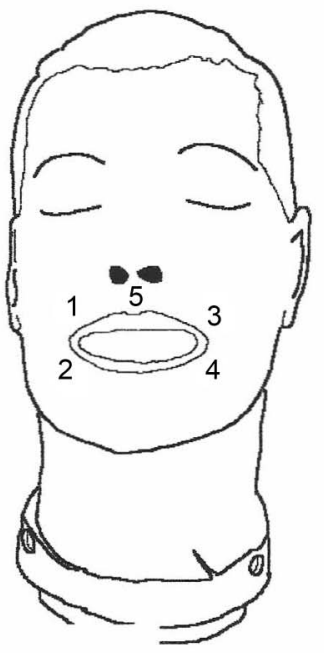

Modified Lillihei

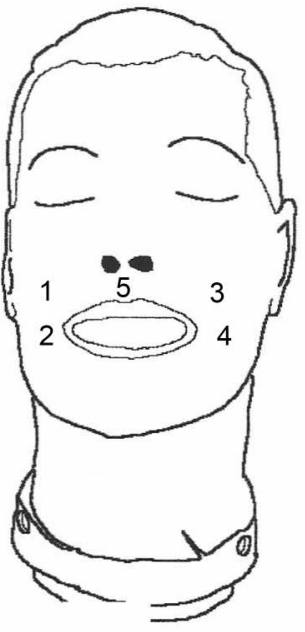

6 Back of neck

Hollister

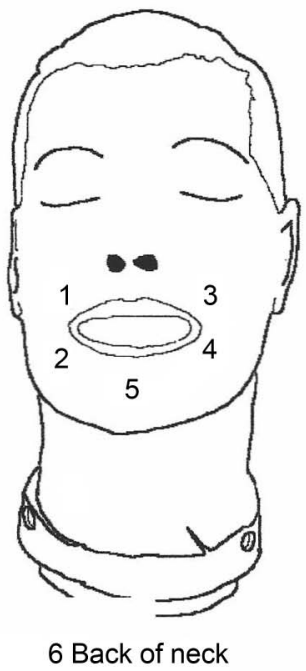

All other devices

Fig. 3. Sensor placement for head rotation study. Numbers indicate sensor placement. Sensor 6 was on the back of neck for those holders that had a strap.

\section{Data Analysis}

Data were checked for normality using the Shapiro-Wilk test. All normally distributed data were expressed as mean \pm SD. Nonparametric data were analyzed using multivariate analysis of variance, with $P<.05$ as significant. Post hoc analysis was performed using the Tukey honest significant difference test. All data analysis was performed using R 3.0.1 statistical software (R Project for Statistical Computing, http://www.r-project.org, Accessed June 9, 2014).

Static Tug Test. The distance moved was collected for each of the 100 trials per device/technique. Tube displacement of $>5 \mathrm{~cm}$ was considered extubation; the remaining successful drops for the holder were evaluated. 


\section{COMPARISON OF ETT HOLDERS}

Table 2. Static Tug Results by Device/Technique

\begin{tabular}{|c|c|c|c|c|c|c|}
\hline Device/Technique & $n$ & Median $(\mathrm{cm})$ & Minimum $(\mathrm{cm})$ & Maximum $(\mathrm{cm})$ & IQR & Type \\
\hline Hollister AnchorFast ETT holder & 80 & 0.00 & 0.00 & 0.10 & $0-0.00$ & Commercial \\
\hline Hy-Tape & 80 & 0.00 & 0.00 & 0.10 & $0-0.00$ & Noncommercial \\
\hline Modified Lillehei method & 80 & 0.00 & 0.00 & 0.10 & $0-0.00$ & Noncommercial \\
\hline Laerdal Medical Thomas tube holder & 80 & 0.00 & 0.00 & 0.20 & $0-0.10$ & Commercial \\
\hline Lillehei method & 80 & 0.00 & 0.00 & 1.10 & $0-0.10$ & Noncommercial \\
\hline Marpac 320 ETT holder with head strap & 80 & 0.00 & 0.00 & 0.50 & $0-0.10$ & Commercial \\
\hline Rolling hitch knot & 80 & 0.00 & 0.00 & 0.30 & $0-0.10$ & Noncommercial \\
\hline Teleflex cushioned ETT holder 1065 & 80 & 0.00 & 0.00 & 0.30 & $0-0.10$ & Commercial \\
\hline Portex Quickstrap ETT holder & 78 & 0.00 & 0.00 & 0.50 & $0-0.20$ & Commercial \\
\hline Clove hitch knot & 80 & 0.10 & 0.00 & 1.00 & $0-0.10$ & Noncommercial \\
\hline Ambu ETT holder (with white strap) 320264041 & 80 & 0.10 & 0.00 & 0.80 & $0-0.12$ & Commercial \\
\hline Ambu ETT holder (with blue strap) 320264040 & 80 & 0.10 & 0.00 & 0.80 & $0-0.20$ & Commercial \\
\hline Cow hitch knot & 80 & 0.10 & 0.00 & 1.40 & $0-0.20$ & Noncommercial \\
\hline Dale Stabilock ETT holder & 80 & 0.10 & 0.00 & 1.10 & $0-0.20$ & Commercial \\
\hline Precision Medical ETT holder PM1110 & 67 & 0.20 & 0.00 & 3.50 & $0-1.05$ & Commercial \\
\hline Marpac 320 ETT holder & 80 & 0.20 & 0.00 & 1.00 & $0.1-0.30$ & Commercial \\
\hline
\end{tabular}

Values are the median distance displaced in the static tug test. $n<80$ indicates the number of complete extubations.

$\mathrm{ETT}=$ endotracheal tube

$\mathrm{IQR}=$ interquartile range

Side-to-Side ETT Movement. The time needed to move the ETT was compared using one-way analysis of variance with the Tukey honest significant difference test between devices and within each device.

Head Rotation Study. The system was allowed to stabilize for $1 \mathrm{~min}$. The next 5 complete cycles were collected, and the voltage readings of the force-sensing resistors were converted into newtons based on calibration curves specific for each of the 6 sensors. If any device or technique did not have a part that went around the neck of the intubation head or that touched a portion of the face such as the chin, those sensors were not included in the analysis.

The vertical axis movement data were collected at $3^{\circ}$, $15^{\circ}$, and $30^{\circ}$ positions. The horizontal axis movement data were collected at $-35,0$, and $35^{\circ}$ positions. Five data points at each of the 3 angles were selected for each device. Since 5 of the same type of ETT holders were used during each evaluation, a total of 25 data points for each angle for each device were analyzed. If 5 data points at a specific angle were not available, the closest data point from the preceding or following angle was recorded to bring the total to 5 . We used repeated-measures multivariate analysis of variance to compare the effects of device, angle of rotation/elevation, type of device (commercial or noncommercial), and the trial run between devices, as well as to compare each device with itself. For all evaluations, $P<.05$ was considered significant.

\section{Results}

\section{Static Tug Test}

There were 1,600 potential observations. During testing, 17 complete dislodgements were noted (2 with the Portex Quickstrap and 15 with the Precision Medical ETT holder PM1110). To compensate for stretching of the new material, the first series of 5 drops for each holder was removed (run series 1). Two of the 17 extubations occurred in this series, both for the PM1110 device, and they were omitted from analysis. This resulted in a total of 1,265/1,280 observations. The median displacement for this group was $0.00 \mathrm{~cm}$ (interquartile range of $0.0-0.10 \mathrm{~cm})$. The holders/techniques with the least movement (median, interquartile range) were the Hollister AnchorFast $(0.0 \mathrm{~cm}, 0.0-0.00 \mathrm{~cm})$, Hy-Tape $(0.0 \mathrm{~cm}$, $0.0-0.00 \mathrm{~cm})$, and the modified Lillehei method $(0.0 \mathrm{~cm}$, $0.0-0.00 \mathrm{~cm})$, and the holder with the greatest displacement was the Precision Medical PM1110 $(0.2 \mathrm{~cm}, 0-1.1 \mathrm{~cm}$, $P<.001$ ) (Table 2). The displacement distance for both commercial and noncommercial devices was the same (median of $0 \mathrm{~cm}$, interquartile range of $0.0-0.10 \mathrm{~cm}$ ), but all of the extubations that occurred during testing happened with the commercial devices, and none occurred with the noncommercial devices $(P=.001)$.

\section{Tube-Repositioning Study}

An average of $13.8 \pm 12.3 \mathrm{~s}$ was required to move the ETT for the 9 devices/methods evaluated. The shortest period of time was documented for the Marpac 320 
Table 3. Time Needed to Move the ETT by Device/Technique

\begin{tabular}{lr}
\hline \hline \multicolumn{1}{c}{ Device/Technique } & Mean \pm SD (s) \\
\hline Marpac 320 ETT holder & $0.82 \pm 0.14$ \\
Hollister AnchorFast ETT holder & $1.25 \pm 0.20$ \\
Cow hitch knot & $2.79 \pm 0.34$ \\
Clove hitch knot & $4.21 \pm 0.54$ \\
Rolling hitch knot & $7.48 \pm 1.16$ \\
Bow knot* & $8.48 \pm 0.83$ \\
Dale Stabilock ETT holder & $14.67 \pm 1.10$ \\
Lillehei method & $20.84 \pm 5.51$ \\
Hy-Tape & $32.02 \pm 4.00$ \\
Modified Lillehei method & $34.42 \pm 3.44$ \\
& \\
\hline Values indicate the time needed to move the ETT from one side of the mouth to the other. \\
* See text for description of knots. \\
ETT = endotracheal tube
\end{tabular}

$(0.82 \pm 0.14 \mathrm{~s})$ and Hollister AnchorFast $(1.25 \pm 0.20 \mathrm{~s})$. The difference between these 2 devices was not significant $(P=.99)$. The 2 slowest methods were Hy-Tape $(32.0 \pm 4 \mathrm{~s})$ and the modified Lillehei method (34.4 $\pm 3.44 \mathrm{~s})$ Again, the difference between these 2 methods was not significant $(P=.51)$ (Table 3). It took less time to move the ETT using commercially available devices compared with noncommercial techniques $(5.58 \pm 6.57 \mathrm{~s}$ vs $17.91 \pm 12.48 \mathrm{~s}, P<.001)$.

\section{Head Rotation Studies}

There were 1,200 observations for sensors 1-4. Both sensors 5 and 6 had 1,050 observations each. Sensor 5 was placed on the chin, and sensor 6 was placed on the back of the neck. This was done because 3 holders have designs that do not cover those sensor areas on a mannequin (Hy-Tape, no sensor 5 or 6; Lillehei method, no sensor 5; modified Lillehei method, no sensor 5, Modified Lillehei method, no force reading on sensor 5 and no sensor 6) (see Fig. 3).

Vertical Head Lift. The forces measured varied for each sensor for all of the ETT holders $(P<.001)$. The greatest force recorded at any time was $3.52 \mathrm{~N}$ (Ambu ETT holder $320264040)$ at sensor 1 . The least force recorded was $<0.2 \mathrm{~N}$; this measured force was beyond the resolution for the sensors, so a more precise measurement is unavailable. Grouping the forces into the 3 angles $\left(3^{\circ}, 15^{\circ}\right.$, and $30^{\circ}$ ), force readings at individual sensors varied among devices: sensors $1-4, P<.001$; sensor $6, P=.002$; and sensor $5, P=.07$. Summary force readings (mean $\pm \mathrm{SD}$ ) at each sensor for each device are provided (see the supplementary materials at http://www.rcjournal.com). Commercial devices exerted more force than noncommercial methods (all sensors, $P<.001$ ) (Fig. 4). Analysis of the individual devices showed that the Ambu ETT holder 320264040, Marpac 320 with a head strap, cow hitch knot, Lillehei method, and Teleflex cushioned ETT holder 1065

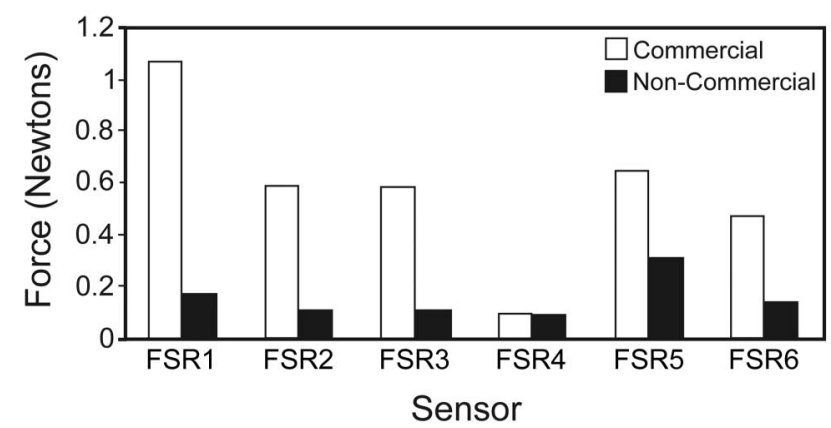

Fig. 4. Graph of pooled forces measured during the vertical head lift at each sensor for commercial and noncommercial ETT holders.

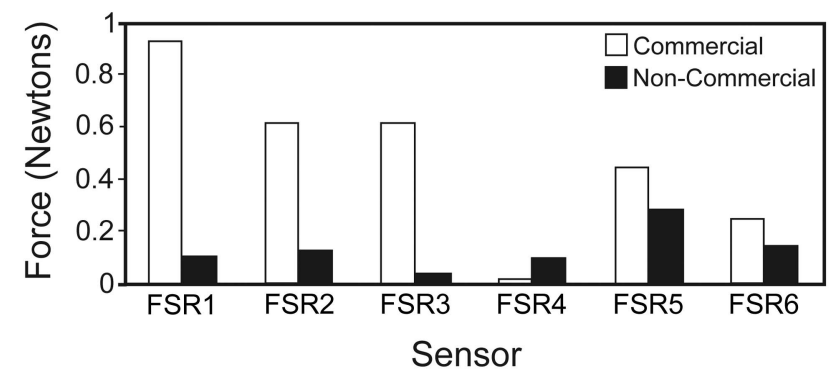

Fig. 5. Graph of pooled forces measured during the horizontal head rotation at each sensor for commercial and noncommercial ETT holders.

did not show any difference between forces measured among the sensors.

Horizontal Head Rotation. Force readings were different for each sensor with every device $(P<.001)$. The greatest force recorded at any time was 3.40 N (Ambu ETT holder 320264040) at sensor 1. The least force recorded was $<0.2 \mathrm{~N}$; this measured force was beyond the resolution for the sensors, so a more precise measurement is unavailable. Grouping the data into the 3 angles $\left(-35^{\circ}\right.$, $0^{\circ}$, and $35^{\circ}$ ), the forces measured for all devices showed a wide variation $(P<.001)$, with the exception of the Lillehei method $(P=.068)$. Summary force readings (mean $\pm \mathrm{SD}$ ) are provided (see the supplementary materials at http://www.rcjournal.com).

Commercially available devices exerted more pressure at all sensors than noncommercial devices, with the exception of sensor 4 . It registered higher forces with noncommercial devices than with commercial devices (Fig. 5).

\section{Discussion}

This is the most comprehensive study to date examining a large variety of ETT-securing methods under 4 different simulated clinical conditions. No single device design performed well in all of the evaluations. 


\section{COMPARISON OF ETT HoldERS}

The force exerted on the patient's face by many of the commercial securing devices may result in discomfort and formation of pressure ulcers. Noncommercial techniques use materials (tape, string) that are more form-fitting to the patient's face and therefore do not have the same pressure point issues as seen with commercial devices.

Barnason et $\mathrm{al}^{8}$ looked prospectively at the impact of 2 securing techniques on patient comfort and skin integrity. The techniques used to secure the airway were the Lillehei method and cotton twill with a cow hitch knot. Pressure on the patient's face was based more on descriptive findings rather than quantifiable measurements. Their conclusion was that both techniques were equally effective in preventing oral mucosal breakdown, which is consistent with our findings.

Resistance to movement after the ETT has been subjected to an unplanned tugging motion is crucial to the function of any ETT holder. In general, because of variation in gripping ability, the noncommercial devices did not perform as well as the commercial devices.

Two separate studies ${ }^{5,6}$ used a technique similar to ours for creating dynamic torque on the ETT by dropping a weight via a pulley. The design of Murdoch and Holdgate ${ }^{6}$ was for the force generated to be perpendicular to the intubation mannequin. They considered a movement of the ETT of $\geq 20 \mathrm{~mm}$ to be a major displacement. The 2 techniques evaluated were the Laerdal Medical Thomas tube holder and twill tape tied with a reef (square) knot. During testing, they noted that in $61 \%$ of the trials, the tape allowed ETT movement of $\geq 20 \mathrm{~mm}$, whereas none of the trials with the Laerdal Medical device met failure criteria. An explanation for this discrepancy may be that the reef knot was a poor choice and that a better holding knot would have been the rolling hitch.

Lovett et al ${ }^{5}$ used a polyvinyl chloride pipe as an intubation model and saturated the holders once applied with saline to simulate oral secretions. They measured the actual force generated when dropping 2.5-, 5-, and 10-pound weights and measured the distance the ETT moved after 6 and 15 drops. This method provided the average distance moved after a sequence of drops rather than the effects of each drop. Although there was a difference in models (polyvinyl chloride pipe versus intubation mannequin), the performance was similar to what we experienced for the 3 holders/techniques that were common to both studies (Lillehei method, Precision Medical ETT holder PM1110, and Thomas tube holder).

The overall poorest performer during the static tug test (Precision Medical ETT holder PM1110) had a thin securing strap, which stretched significantly. It is the elasticity of the strap that allowed for a large displacement of the ETT. This finding is similar to that of Carlson et al. ${ }^{9} \mathrm{In}$ fact, the strap was so compliant that it was difficult to secure the airway with only a 2-finger tight assessment, with 2 fingers fitting snugly between the face of the mannequin and the securing strap. This issue was evident to some degree with other devices employing a similar design, where there was too much material, requiring multiple layers of wrapping or, in extreme conditions, tightening beyond the recommended 2-finger test. Devices in this class were the Ambu ETT holder with a blue silicone strap, Smiths Medical Portex Quickstrap, and Dale Stabilock.

Several manufacturers secure an ETT by compressing it between a clamp or screw. These mechanisms have an influence on the cross-sectional shape of the airway. This distortion may have an influence on airway resistance, but it was not within the scope of this investigation to measure airway resistance.

Several commercial devices are designed to allow for quick tube relocation, and in fact, they outperformed the noncommercial devices. A common design feature for the commercially prepared holders is a track where the ETT can be guided from one point to another. The noncommercial holders all require disassembly and reassembly of the securing technique; 2 techniques (modified Lillehei method and Hy-Tape) require a completely new holder to be fashioned after moving the airway. This results in the airway being unsecured and susceptible to displacement either by the practitioner or by the patient.

\section{Causes of Unplanned Extubation}

There are 2 classifications of unplanned extubation: patient-initiated and practitioner-initiated. Both categories of unplanned extubation place the patient at some risk depending upon the clinical status. It has been one of the primary goals of ETT fixation to secure the ETT in a manner in which the airway is unlikely to become dislodged yet is flexible enough not to cause damage to surrounding tissue. In one multi-center study examining unplanned extubation, the lack of a strong fixation device was found to be one of the risk factors identified. ${ }^{10}$

Unplanned extubation can lead to comorbidity, including increased ventilator days, and increased mortality ${ }^{10,11}$ and has been associated with an increased likelihood of transfer to chronic care facilities. ${ }^{11}$ Among the factors affecting transfer are skin integrity, complexity of the ETT-securing device, oral care, speed of application of the device to the ETT, and patient comfort. No one device can address every factor, nor is it possible to test for every contingency.

\section{Ability to Relocate the ETT}

Devices with an integrated bite block prevent the ETT from being repositioned, which may lead to pressure ulcers in the mouth and surrounding tissue. Kuhn et al ${ }^{12}$ reported the development of a necrotic region on the tongue of a patient after only $8 \mathrm{~h}$ of intubation. The bite block incorporated with some devices can also interfere with adequate oral hygiene by preventing access to the oral 


\section{COMPARISON OF ETT HoldERS}

cavity. There is at least one report of an added bite block interfering with cuff function. ${ }^{13}$

\section{Limitations}

There are several limitations to this study. First, this was a bench study, and the surface of the mannequin may have behaved differently with the various adhesives used by some of the manufacturers than natural skin. This property was standardized with the application of the Blenderm product whenever the adhesive properties were being stressed during the static tug. Nonetheless, the skin of the intubation head does not have the same tensile qualities of real skin and thus may have impacted the pressure readings measured when securing the ETT.

Second, all readings were done at ambient temperature $\left(\sim 20^{\circ} \mathrm{C}\right)$ without oral secretions saturating the securing devices/methods. Previous studies examining the securing properties of various devices both in simulation and in vitro identified oral secretions as factors that affect function. ${ }^{5,8,9,14}$ These studies either had a small sample size or were anecdotal discussions of various ETT holders. In the study by Carlson et al, ${ }^{9}$ extubation force was determined using $<24$-h-old refrigerated cadavers, and actual force was measured using a strain gauge. The studies by both Arrott and Talley ${ }^{15}$ and Barnason et $\mathrm{al}^{8}$ were either descriptive or observational in nature. In clinical practice, there is no opportunity to pre-stress the neck strap, as occurred during the static tug test, so actual ETT displacement distances may be greater than reported here.

Third, during the tube movement, the goal was to move the airway as quickly as possible. It is likely that under clinical circumstances, concern for the safety of the patient would have a slowing effect on tube movement. As a result, timing was most likely shorter than during actual clinical conditions.

Fourth, movement of the ETT farther into the airway, that is the main bronchus, was not evaluated in this study. This is an important issue and should be considered for future studies.

Finally, this study was not designed to measure the convenience factor in application of the devices, which can have an impact on choice of device/method.

\section{Conclusions}

The conclusions of this study are as follows: (1) noncommercial airway holders exert less force on a patient's face than commercial devices; (2) airway stability is affected by the type of securing method selected; and (3) many of the commercial securing devices allow for rapid but secure movement of the artificial airway from one side of the mouth to the other. However, at this time, there is no ideal device or method for securing an ETT.

\section{REFERENCES}

1. da Silva PS, Fonseca MC. Unplanned endotracheal extubations in the intensive care unit. Anesth Analg 2012;114(5):1003-1014.

2. Zaratkiewicz S, Teegardin C, Whitney JD. Retrospective review of the reduction of oral pressure ulcers in mechanically ventilated patients: a change in practice. Crit Care Nurs Q 2012;35(3):247-254.

3. American Heart Association. Part 7.1: adjuncts for airway control and ventilation. Circulation 2005;112(24 suppl):IV-51-IV-57.

4. Pitts R, Fisher D, Sulemanji D, Kratohvil J, Jiang Y, Kacmarek R. Variables affecting leakage past endotracheal tube cuffs: a bench study. Intensive Care Med 2010;36(12):2066-2073.

5. Lovett PB, Flaxman A, Stürmann KM, Bijur P. The insecure airway: a comparison of knots and commercial devices for securing endotracheal tubes. BMC Emerg Med 2006;6(1):7.

6. Murdoch E, Holdgate A. A comparison of tape-tying versus a tube holding device for securing endotracheal tubes in adults. Anaesth Intensive Care 2007;35(5):730-735.

7. Dodek P, Keenan S, Cook D, Heyland D, Jacka M, Hand L, et al. Evidence-based clinical practice guideline for the prevention of ventilator-associated pneumonia. Ann Intern Med 2004;141(4): 305-313.

8. Barnason S, Graham J, Wild MC, Jensen LB, Rasmussen D, Schulz $\mathrm{P}$, et al. Comparison of two endotracheal tube securement techniques on unplanned extubation, oral mucosa, and facial skin integrity. Heart Lung 1998;27(6):409-417.

9. Carlson J, Mayrose J, Krause R, Jehle D. Extubation force: tape versus endotracheal tube holders. Ann Emerg Med 2007;50(6): 686-691.

10. Boulain T. Unplanned extubations in the adult intensive care unit: a prospective multicenter study. Association des Reanimateurs du Centre-Ouest. Am J Respir Crit Care Med 1998;157(4 Pt 1):11311137.

11. Epstein SK, Nevins ML, Chung J. Effect of unplanned extubation on outcome of mechanical ventilation. Am J Respir Crit Care Med 2000;161(6): 1912-1916.

12. Kuhn MA, Zeitler DM, Myssiorek DJ. Tongue necrosis: a rare complication of oral intubation. Laryngoscope 2010;120(Suppl 4):S159.

13. Adams JR, Hoffman J, Lavelle J, Mireles-Cabodevila E. Pilot balloon malfunction caused by endotracheal tube bite blockers. Respir Care 2014;59(2):e22-e24.

14. Levy H, Griego L. A comparative study of oral endotracheal tube securing methods. Chest 1993;104(5):1537-1540.

15. Arrott JJ, Talley AW. Endotracheal tube holder. Anesth Analg 1974;53(1):70-71.

This article is approved for Continuing Respiratory Care Education credit. For information and to obtain your CRCE

(free to AARC members) visit www.rcjournal.com

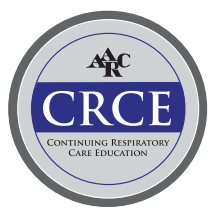

